\title{
Phase Segregation of Medial Septal GABAergic Neurons during Hippocampal Theta Activity
}

\author{
Zsolt Borhegyi, ${ }^{1 \star}$ Viktor Varga, ${ }^{1 *}$ Nóra Szilágyi, ${ }^{2}$ Dániel Fabo, ${ }^{1}$ and Tamás F. Freund ${ }^{1}$ \\ ${ }^{1}$ Department of Cellular and Network Neurobiology, Institute of Experimental Medicine of the Hungarian Academy of Science, Budapest 1083, Hungary, \\ and ${ }^{2}$ Department of Physiology and Neurobiology, Eötvös Loránd University of Sciences, Budapest 1117, Hungary
}

Septo-hippocampal GABAergic neurons immunoreactive for parvalbumin are thought to play a crucial role in the generation of hippocampal theta oscillations associated with a specific stage of memory formation. Here we use in vivo juxtacellular recording and filling in the medial septum followed by immunocytochemical identification of the recorded cells containing parvalbumin to determine their firing pattern, phase relationship with hippocampal theta, morphology, and to thereby reveal their involvement in the generation of hippocampal theta activity. We have demonstrated that GABAergic medial septal neurons form two distinct populations exhibiting highly regular bursting activity that is tightly coupled to either the trough $\left(178^{\circ}\right)$ or the peak $\left(330^{\circ}\right)$ of hippocampal theta waves. Additionally, different types of bursting as well as nonbursting activity patterns were also observed. The morphological reconstruction of theta-bursting neurons revealed extensive axon arbors of these cells with numerous local collaterals establishing symmetrical synapses; thus, synchrony among the septal pacemaker units may be brought about by their recurrent collateral interactions. Long projecting axons could also be found running dorsally toward the hippocampus and ventrally in the direction of basal forebrain regions. We conclude that GABAergic neurons in the medial septum, which are known to selectively innervate hippocampal interneurons, are in a position to induce rhythmic disinhibition in the hippocampus and other theta-related subcortical areas at two different phases of hippocampal theta.

Key words: EEG; juxtacellular; wavelet; parvalbumin; burst; immunocytochemistry

\section{Introduction}

Decades of research has pointed to the hippocampus as a key structure in memory formation (Squire, 1992). Different stages of memory formation may be linked to the two major behaviordependent hippocampal activity patterns, large-amplitude irregular activity with sharp waves and synchronous oscillation at theta frequency (Buzsaki et al., 1983). Hippocampal network activity is under the control of subcortical regions, of which the medial septum (MS) has a pivotal role in the generation of theta oscillations (Vertes and Kocsis, 1997).

The medial septum establishes reciprocal connections with the hippocampus (Toth et al., 1993). The septo-hippocampal pathway is formed by cholinergic (Frotscher and Leranth, 1985) and parvalbumin-immunoreactive (PV-IR) GABAergic neurons of the medial septum (Freund, 1989), and the latter are known to selectively innervate GABAergic interneurons in the hippocampus (Freund and Antal, 1988).

Received April 15, 2004; revised July 8, 2004; accepted July 28, 2004.

This work was supported by National Institutes of Health Grant MH 54671, the Howard Hughes Medical Institute, Országos Tudományos Kutatási Alapprogramok Grants T-32251 and T-034638, and a Bolyai Scholarship (Z.B.). We gratefully thank Drs. G. Buzsaki, X. J. Wang, I. Mody, and B. Kocsis for the critical reading of this manuscript and Dr. Mark D. Eyre for proofreading it. We also thank Katalin Lengyel, Emöke Simon, Bertalan Henter, and Gyözö Goda for technical help.

*Z.B. and V.V. contributed equally to this work.

Correspondence should be addressed to Tamás F. Freund, Institute of Experimental Medicine, Hungarian Academy of Science, Szigony utca 43, Budapest 1083, Hungary. E-mail: freund@koki.hu.

DOI:10.1523/JNEUROSCI.1413-04.2004

Copyright $\odot 2004$ Society for Neuroscience $\quad$ 0270-6474/04/248470-10\$15.00/0
Numerous attempts have been made to characterize the basic physiological parameters of GABAergic, PV-IR neurons of the MS in vitro. These cells recorded in septal slices fired highfrequency non-adapting spike trains in response to a depolarizing current injection (Morris et al., 1999). In the living animal, the majority of MS neurons show hippocampal theta-related firing (Gogolak et al., 1968) at various patterns: theta bursting, nonbursting theta-modulated, and theta-independent firing (Gaztelu and Buno, 1982). Based on intracellularly recorded spike shape and firing pattern, burst-firing, fast-spiking neurons with short-duration action potentials were hypothesized to be GABAergic neurons (Brazhnik and Fox, 1999). By analyzing septo-hippocampal interactions, strong phase coupling was observed between medial septal unit activity and hippocampal theta in both anesthetized and freely moving animals (King et al., 1998). Furthermore, putative GABAergic cells were shown to be inhibited during hippocampal sharp waves (Dragoi et al., 1999).

However, no information is yet available in the literature about the correlation between the anatomical-neurochemical identity of medial septal cells and their in vivo firing properties related to hippocampal theta. This information is fundamental for the understanding of how septal pacemaker activity is able to generate theta oscillations in the hippocampus. It remains to be established whether the GABAergic medial septal neurons fire rhythmic bursts and whether these are locked to specific phases of hippocampal theta. Furthermore, no morphological data are available about identified GABAergic medial septal neurons with rhythmic burst firing, which would help to explain how the indi- 
vidual bursting units can produce a coherent, rhythmic output of the medial septal network.

In the present study, we used an approach that allows us to answer several of these complex questions by the unique simultaneous identification of relevant physiological and anatomical features of medial septal neurons, namely (1) the in vivo firing characteristics (e.g., rhythmic bursting), (2) phase relationship in firing to hippocampal theta, (3) the neurochemical identity (PV positive, GABAergic or PV negative), and (4) the precise location and morphology of the soma dendritic and axonal arbors, together with the postsynaptic elements.

\section{Materials and Methods Surgery}

Rats were anesthetized with intraperitoneally administered $40 \%$ urethane (dose, $0.37 \mathrm{ml} / 100 \mathrm{gm}$ ). A homeothermic heating pad connected to a rectal probe held body temperature constant $\left(36^{\circ} \mathrm{C}\right)$. Experiments were performed according to the guidelines of the Institutional Ethical Codex and the Hungarian Act of Animal Care and Experimentation (1998, XXVIII, section 243/1998), which is in full agreement with the regulation of animal experiments in the European Union. All efforts were made to minimize pain and suffering and to reduce the number of animals used.

\section{Electrophysiological recording}

A small window was drilled over the medial septal region, and a hole was drilled over the right hippocampus. Hippocampal EEG was recorded by monopolar tungsten microelectrodes (in vitro impedance, $0.8-3 \mathrm{M} \Omega$ ) lowered into the pyramidal layer of the CA1 region by a mechanical microdrive (Hugo Sachs Elektronik, March-Hugstetten, Germany). During the descent of the electrode, multiunit activity was monitored. An abrupt increase in multiunit activity and the appearance of complex spikes between 1.9 and $2.2 \mathrm{~mm}$ from the brain surface were used as landmarks for the pyramidal layer. The recorded signal was amplified, bandpass filtered $(0.3 \mathrm{~Hz}$ to $5 \mathrm{kHz}$ or, in some cases, $0.3-150 \mathrm{~Hz}$; BioAmp; SuperTech, Budapest, Hungary) and digitized at $10 \mathrm{kHz}$ (micro1401 mkII; Cambridge Electronics Design, Cambridge, UK). Septal units were recorded by glass microelectrodes (in vivo impedance, $20-40$ $\mathrm{M} \Omega$ ) pulled from borosilicate glass capillaries (1.5 $\mathrm{mm}$ outer diameter, $0.86 \mathrm{~mm}$ inner diameter; Sutter Instruments, Novato, CA) and filled with $0.5 \mathrm{M} \mathrm{NaCl}$ and $3 \%$ biocytin (Sigma, St. Louis, MO) or 2\% Neurobiotin (Vector Laboratories, Burlingame, CA). The electrode was lowered into the brain by a piezoelectric microdrive (6000 ULN; Burleigh Instruments, Fishers, NY). Neuronal signals were amplified by a DC amplifier (Axoclamp 2B; Axon Instruments, Foster City, CA), filtered between 0.1 and $5 \mathrm{kHz}$ by a signal conditioner (SuperTech), and digitized at $10 \mathrm{kHz}$ (Cambridge Electronics Design). Hippocampal theta occurred spontaneously and/or was elicited by tail pinch. Recording was followed by the juxtacellular labeling of the neuron (Pinault, 1996). Briefly, a positive injection current was applied through the electrode in a $200 \mathrm{msec}$ on-off duty cycle on top of a low-intensity negative DC $(0.4-1.5 \mathrm{nA})$ while the activity of the neuron was continuously monitored. Injection current intensity was increased until the modulation of neuronal firing (up to 10 $\mathrm{nA}$ ) occurred. The length of labeling was 5-10 min.

\section{Data analysis}

Wavelet analysis. The EEG was analyzed in the time-frequency domain by wavelet decomposition on 101 or 141 scales from 1.5 to $193.6 \mathrm{~Hz}$. The lower-resolution wavelet transform was used for the selection of EEG segments with defined power density in the theta band. For all other wavelet-based calculation, the high-resolution decomposition was used. The continuous wavelet transform of the sampled time series $x_{n}$ is a convolution of it with the wavelet function $\Psi$ :

$$
W_{n}(s)=\sum_{n^{\prime}=0}^{N-1} x_{n^{\prime}} \Psi *\left[\frac{\left(n^{\prime}-n\right) \delta t}{s}\right],
$$

where ${ }^{\star}$ is the complex conjugate.
For the calculation of the wavelet power spectrum, the Morlet wavelet was used (Muthuswamy and Thakor, 1998):

$$
\Psi_{0}(\eta)=\pi^{-1 / 4} e^{i \omega_{o} \eta} e^{-\eta^{2 / 2}}
$$

The cross-wavelet spectrum for the time series $X$ and $Y$ is as follows:

$$
W_{n}^{X Y}(s)=W_{n}^{X}(s) W_{n}^{Y^{*}}(s),
$$

where ${ }^{*}$ is the complex conjugate.

Selection of analyzed segments, EEG, and spike train pre-processing. Before wavelet decomposition, the EEG was resampled at 500 or $400 \mathrm{~Hz}$ (for segment selection or cross-wavelet calculation, respectively). Theta band was defined between 3 and $6 \mathrm{~Hz}$ considering the downward shift of theta frequency range under urethane anesthesia (Leung, 1985). Two EEG segments were selected for additional analysis based on wavelet decomposition: (1) baseline (low theta), with $<50 \%$ power in the theta range and no or less than three ripples and (2) tail pinch (high theta), with $>50 \%$ relative power in the theta range. The minimal length of analyzed segments was $5 \mathrm{sec}$. For the detection of ripples, the EEG was filtered between 90 and $145 \mathrm{~Hz}$ (Klausberger et al., 2003) by high-order finite impulse response filters, and the root mean square (RMS) of the filtered signal was calculated in 100-point non-overlapping windows. Ripple detection threshold was mean +5 SD of the RMS of the signal, and the beginning and end of ripples were determined at RMS values of mean + $1 \mathrm{SD}$. For the analysis of neuronal firing pattern, unit recordings were amplitude discriminated. Firing rate was calculated for the selected (baseline, tail pinch, and ripple) recording periods. When comparing baseline and tail-pinch firing rate, a $>50 \%$ decrease or increase was termed as inhibition or activation, respectively. In baseline versus ripple comparison, inhibition or activation has to be detected in at least $>50 \%$ of ripples in the bin overlapping with the given ripple. Additionally, spike count during ripples was compared with that of neighboring, ripple-long segments by Mann-Whitney $U$ test ( $p=0.05$ ).

Firing pattern analysis. To detect theta-modulated activity of the unit time series ( spike $=1$ interspike data points $=0$ ), conventional fast Fourier transformation (FFT) was used. In this calculation, time resolution was not important, but, for obtaining the highest possible frequency resolution, FFT was preferred over wavelet decomposition. The discriminated unit events were converted into a spike density function (SDF) (Szucs, 1998) by convolution with a 1000-point Blackman-Harris window. In the SDF, amplitude is proportional to instantaneous frequency and, by choosing appropriate window length, fine details of the spike train can be maintained without having abrupt amplitude jumps (like action potentials). Subsequently, the relative power in the theta band of the unit signal was calculated from the spectral density function using the highest possible resolution ( 0.15 or $0.3 \mathrm{~Hz}$ depending on the length of the analyzed segment) and given as percentage power in the theta band.

Burst identification and analysis. The structure of the spike train was investigated by separating spike clusters occurring periodically in correlation with the hippocampal theta. The separated spike clusters are named here as theta bursts. Theta bursts were separated by the onedimensional cluster analysis of interspike intervals (for details on cluster analysis, see below). It is known that the interspike interval histogram of periodically bursting neurons is bimodal (Cocatre-Zilgien and Delcomyn, 1992). By plotting the interspike intervals on a recurrence plot (Poincaré map) intraburst and interburst intervals appear as separable interval clusters (Dekhuijzen and Bagust, 1996). Based on this observation, bursts were separated by the cluster analysis of interspike intervals. Euclidean distance and Ward's amalgamation rule was used in the cluster analysis. Three to seven clusters were generated by cutting the icicle plot (dendrogram) at different linkage distances. The biggest cluster containing the smallest interval was selected in each case (three to seven clusters) assuming that the intraburst (small) intervals outnumber interburst intervals. In the next step, the distribution of intervals in the five selected clusters (the biggest cluster in each "cut" of the dendrogram) was visually compared with the interspike interval histogram of all spikes. Intervals of the cluster, which overlapped with the first peak of the bimodal interspike interval histogram, were selected as intraburst intervals. Finally, based on the location of intraburst intervals, the bursts were marked in 
the time series. The calculated burst parameters were as follows: burstiness (proportion of spikes in bursts), intraburst frequency, number of spikes in bursts, and burst length. For the comparison of firing rate, relative theta power, and burst parameters, the Mann-Whitney $U$ test was used with a level of significance set at $p<0.05$.

EEG-unit correlation and phase relationship analysis. The intensity of theta activity allowed us to analyze all but two bursting PV-IR neurons. The phase was calculated independently from other physiological parameters. For analyzing the interaction between the septal unit and the hippocampal EEG, the wavelet cross-spectrum of the two signals was computed (for details on wavelet transform, see the first paragraph of "Data analysis"). First, the unit time series was converted to a continuous signal by convolving it with a sinc function $(\sin (x) / x)$ and then resampled at $400 \mathrm{~Hz}$. The cross-wavelet spectra were calculated between the wavelet spectrum of the converted unit signal and the downsampled EEG (from $10 \mathrm{kHz}$ to $400 \mathrm{~Hz}$ ). Phase synchronization was reflected in phase angle preferences of the cross-wavelet phases. Relative phase distribution of the EEG and unit activity for the theta band was obtained from the crosswavelet phase (phase difference between the EEG and the converted unit train). As an exact phase synchrony measure, the circular mean resultant length was used. It was estimated from the angular distribution of the cross-wavelet phase in the theta band and termed as the synchronicity index (one-circular variance; range: 0 , no phase synchrony; 1 , exact phase synchrony). Only contributions from phases belonging to $50 \%$ thresholded wavelet theta power (relative to total power) as noise filtered coincident events were kept. Circular descriptive and inferential statistics were used to test several hypotheses on the distribution of PV-IR phase angles as described below. Significant deviation from random (uniform) angular distribution along the circle indicates directionality. Rao's spacing test (Rao, 1976) was performed to test the uniformicity null hypothesis. The probability distribution [probability distribution function (PDF)] of the relative phase was modeled by a mixture of adequate number of von Mises PDFs. Goodness-of-fit was measured by Bhattacharyya metric (Bhattacharyya, 1943). After the identification of the phase preference groups, the first moment of the relative phase distribution (mean angle) was calculated separately for the groups. Mean phase angles were compared with a hypothetical parameter value by applying Mardia's test (Mardia and Jupp, 2000). Decisions about the difference between mean angles were made by the aid of confidence intervals (Batschelet, 1981) and Watson's $F$ test (Zar, 1996). The concentration parameter $\kappa$ (function of the mean resultant length) for the different angular clusters was also tested to prove a strong tendency of angular preference (Mardia and Jupp, 2000). For generating the firing probability histograms, individual histograms of each cell were averaged bin by bin. Because the number of action potentials used for the generation of individual histograms varied from cell to cell, histograms were normalized before averaging by dividing each bin by the number of reference events.

All analysis except the comparison of firing rate and firing pattern variables was implemented in the Matlab (MathWorks, Natick, MA) environment by self-devised scripts. For statistical comparisons, Statistica (Statsoft, Tulsa, OK) was used. Values are given as mean \pm SD or median \pm interquartile range (if the variable distribution was not normal). To test normality, Kolmogorov-Smirnov and Shapiro-Wilk tests were used.

\section{Anatomy}

After the physiological recording, labeling, and a survival period (10 min to $6 \mathrm{hr}$ ), the animals were perfused transcardially with saline (1 $\mathrm{min})$, followed by $300 \mathrm{ml}$ of fixative containing $4 \%$ paraformaldehyde, $0.05 \%$ glutaraldehyde, and $15 \%$ picric acid in $0.1 \mathrm{~m}$ phosphate buffer (PB). The tissue block containing the septum was sectioned $(60 \mu \mathrm{m})$ and cut on a Vibrotome for immunocytochemistry.

After washes in PB (three times for 20 min each) the sections were transferred into Tris (Sigma)-buffered saline (TBS), pH 7.4, and, from this step, all of the washes and serum dilutions were performed in TBS [0.02\% saponin (Sigma) was added to antibody solutions to enhance penetration]. The sections were incubated with streptavidin-conjugated 7-amino-4-methylcoumarin-3-acetic acid (AMCA) or FITC (1:500; Jackson ImmunoResearch, West Grove, PA) or streptavidin-conjugated
Alexa 488 (1:3000; Molecular Probes, Eugene, OR) for 2 hr to identify the biocytin (Sigma) or Neurobiotin (Vector Laboratories)-labeled neuron by fluorescent microscopy (Axioscope; Zeiss, Oberkochen, Germany) [the wavelength for filter sets absorption and emission (in $\mathrm{nm}$ ): 365 bandpass/420-460, 450-490/512-542, $546 \pm 12 / 590$ long pass]. The section containing the labeled cell was incubated with a mouse antiparvalbumin antibody (PV, 1:1000; Swant, Bellinzona, Switzerland) overnight at room temperature, followed by a secondary antibody [FITC-conjugated goat anti-mouse or AMCA-conjugated donkey antimouse (1:50; Jackson ImmunoResearch) or Cy3-conjugated donkey anti-mouse (1:200; Molecular Probes)] for $2 \mathrm{hr}$.

The PV content of the neuron was documented by digital camera (DP-70; Olympus Optical, Tokyo, Japan), and all sections were incubated with an ABC kit (1:200; Vector Laboratories) for $2 \mathrm{hr}$. The neuron and its processes were visualized by peroxidase reaction using nickel intensified DAB- $4 \mathrm{HCl}$ (Sigma) as chromogen. For electron microscopy, the sections were treated with $\mathrm{OsO}_{4}(1 \%$ in phosphate buffer) for $30 \mathrm{~min}$, dehydrated in an ascending ethanol series (the $70 \%$ ethanol contained $1 \%$ uranyl acetate for $40 \mathrm{~min}$ ) and in propylene oxide, and then embedded in durcupan (Durcupan ACM; Fluka, Neu-Ulm, Germany). Neurons were reconstructed with the help of a camera lucida, and then samples from the cells were reembedded for electron microscopy. The drawings, digitized images taken by the fluorescent- or light-microscope, as well as the scanned electron microscopic negatives were further processed by Adobe Photoshop 7.0 (Adobe Systems, San Jose, CA).

The soma sizes were estimated by using camera lucida drawing of the soma. The outlines were digitized, and the major and minor axes and the area were measured by Scion Image (Scion, Frederick, MD). The distance from the midline was estimated based on the drawings taken from the sections that contained the soma. Morphological parameters (soma area, minor and major axes, and distance from midline) were grouped by using firing pattern [constitutively bursting, phasic ON (tail-pinchresponsive bursting), and tonic ON (tail-pinch-responsive nonbursting)] or phase (trough or peak preference) as grouping variable, and the resulting groups were compared by using Mann-Whitney $U$ test with level of significance set at $p=0.05$.

\section{Results Physiology}

Electrophysiological properties of PV-IR neurons

In the present experiments, a total of 57 recorded neurons were successfully labeled and recovered. Of these, 20 were unequivocally identified as PV-IR (for details, see "Anatomy" below).

All PV-IR neurons changed their firing pattern in response to tail pinch (evoked theta), allowing a classification based on their firing rate and firing pattern. The data are summarized in Tables 1 and 2, and the definitions of firing properties are given in Materials and Methods.

Constitutively bursting neurons. Constitutively bursting neurons $(n=8)$ were characterized by persistent theta bursting activity during both baseline and tail-pinch recording periods (for a representative example, see Fig. $1 A$, and for identification of the neurons, see Fig. 2). However, in response to tail pinch, the level of theta modulation increased because of a larger proportion of intraburst spikes $(88-96 \%)$ and the reduction of burst length leading to higher intraburst frequency compared with the baseline period. Significant elevation of firing rate could be detected in five cells, whereas the remaining three neurons did not change their firing rate significantly. The high value of the synchronicity index $(>0.8)$ indicated an increase of EEG-unit correlation.

The number of hippocampal EEG ripples (minimum of seven episodes) was sufficient for the analysis in the case of three constitutively bursting cells: two of them were inhibited, and one did not exhibit any significant firing rate change (the inhibitory response is demonstrated in Fig. $1 B$ ). In the inhibited cells in this 
Table 1. Firing pattern characteristics of identified neurons

\begin{tabular}{|c|c|c|c|c|c|c|c|c|}
\hline \multirow{2}{*}{ Group } & & \multicolumn{4}{|l|}{ Firing rate $(\mathrm{Hz})$} & & \multicolumn{2}{|l|}{ Theta modulation (\%) } \\
\hline & & $B$ & 1 & & $\mathrm{R}$ & & $B$ & TP \\
\hline \multirow{3}{*}{\multicolumn{2}{|c|}{$\begin{array}{l}\text { CB }(n=8) \\
\text { PON }(n=10) \\
\text { TON }(n=2)\end{array}$}} & $9.29 \pm 3.86$ & \multicolumn{2}{|c|}{$16.52 \pm 9.77$} & $2.6 \pm 4.5$ & & $36.89 \pm 13.03$ & $55.73 \pm 21.84$ \\
\hline & & $9.70 \pm 5.86$ & \multicolumn{2}{|c|}{$16.63 \pm 10.69$} & $18 \pm 24.76$ & & $23.86 \pm 7.48$ & $52.35 \pm 18.87$ \\
\hline & & $8.08 \pm 2.51$ & \multicolumn{2}{|c|}{$21.32 \pm 9.73$} & 6.86 & & $18 \pm 0.68$ & $24.22 \pm 3.56$ \\
\hline & \multicolumn{2}{|c|}{ Burstiness (\%) } & \multicolumn{2}{|c|}{ Intraburst frequency $(\mathrm{Hz})$} & \multicolumn{2}{|l|}{ Spikes in burst } & \multicolumn{2}{|l|}{ Burst length (msec) } \\
\hline & B & TP & B & TP & B & TP & B & TP \\
\hline $\begin{array}{l}\text { CB } \\
\text { PON }\end{array}$ & $88 \pm 14$ & $\begin{array}{l}96 \pm 3 \\
88 \pm 10\end{array}$ & $31.02 \pm 25.14$ & $\begin{array}{l}42.31 \pm 52.7 \\
56.14 \pm 63.1\end{array}$ & $4.13 \pm 1.1$ & $\begin{array}{l}4.09 \pm 2.3 \\
4.99 \pm 2.6\end{array}$ & $104.52 \pm 54.73$ & $\begin{array}{r}76.92 \pm 37.6 \\
87.4 \pm 62.1\end{array}$ \\
\hline
\end{tabular}

Bold indicates median \pm interquartile range values. In all other cases, mean \pm SD are given. CB, Constitutively bursting; PON, phasic ON (tail-pinch-responsive bursting); TON, tonic ON (tail-pinch-responsive nonbursting); B, baseline; TP, tail pinch; $R$, ripple.

Table 2. Phase and unit-EEG synchrony of identified neurons

\begin{tabular}{lll}
\hline Cell code & Phase (degrees) & Synchronicity \\
\hline Trough preference & & \\
137n1 & 166.48 & 0.97 \\
209n1 & 201.10 & 0.95 \\
233n2 & 179.65 & 0.95 \\
$260 \mathrm{n} 1$ & 215.35 & 0.93 \\
$272 \mathrm{n} 1$ & 188.32 & 0.97 \\
$318 \mathrm{n} 1$ & 161.57 & 0.95 \\
$357 \mathrm{n} 2$ & 180.19 & 0.92 \\
359n1 & 135.78 & 0.98 \\
Peak preference & & \\
123n3 & 341.36 & 0.97 \\
250n1 & 350.60 & 0.98 \\
258n2 & 316.36 & 0.89 \\
268n3 & 272.81 & 0.98 \\
306n2 & 326.28 & 0.87 \\
314n1 & 317.02 & 0.98 \\
350n2 & 347.18 & 0.97 \\
351n1 & 348.45 & 0.92 \\
Nonbursting (tonic theta 0N) & & \\
302n2 & 149.25 & 0.90 \\
359n2 & NA & 0.51 \\
\hline
\end{tabular}

Phase is given as mean phase angle; synchronicity is one-circular variance, and NA means that no phase preference could be detected, i.e., the phase histogram of the neuron was uniform.

and in the next group, the number of spikes during the ripple period was significantly lower than that during the preceding and following ripple-long segments $(p<0.05$; Mann-Whitney $U$ test). It should also be noted that inhibition always started before and lasted longer than the defined boundaries (for details, see Materials and Methods) of the corresponding ripple for all inhibited neurons (in this and in the next group).

Tail-pinch-responsive bursting neurons. Tail-pinch-responsive bursting neurons $(n=10)$ showed irregular, non-theta-bursting activity, with no or low level of theta modulation during the baseline recording period, when there was no or a very low level of theta activity in the hippocampus. Theta modulation during the baseline recording period was significantly lower in this group than in the constitutively bursting neurons $(p<0.05$; Mann-Whitney $U$ test). Tail pinch changed their activity to theta-modulated burst firing accompanied by a $>50 \%$ increase in firing frequency in 7 of 10 neurons (for a representative example, see Fig. $1 A$ ).

In this group, activity during ripples was investigated in the case of five cells. One was activated (Fig. $1 B$ ), two were inhibited, and two did not show any change in activity. The elevation in the number of spikes during ripples has not reached the level of significance relative to the neighboring segments. Activation started before and lasted longer than the duration of ripples, and the inhibitory response in both bursting PV-IR groups was more focused than the activation.

Tonic theta ON neurons. Both neurons $(n=2)$ were recorded in experiments in which tail-pinch-responsive bursting or constitutively bursting neurons were also found (for an example, see Fig. $1 A$ ). Either in baseline or in response to tail pinch, these neurons were characterized by a low or moderate level of theta modulation and by non-bursting activity. Tail pinch resulted in a robust increase of firing rate, although no change in firing pattern could be observed. However, EEG-unit correlation increased during tail pinch compared with baseline.

The required number of ripples (see Materials and Methods) could only be found in one cell (the theta-modulated one), showing no significant ripple-associated activity change.

Burst characteristics of PV-IR neurons. More than one-half (10 of $18,56 \%$ ) of the PV-IR neurons fired bursts with $<45 \mathrm{~Hz}$ mean intraburst frequency with low variability, in contrast to the rest of PV-IR neurons in which the lowest mean intraburst frequency was $57 \mathrm{~Hz}$, and high-frequency (>100 Hz) intraburst transients frequently occurred (for details, see Fig. 3, Table 1). Consequently, the variability of intraburst frequencies was high in this group. Moreover, a strong correlation of mean intraburst frequency and variability (measured as SD) could be detected $(r=$ $0.94 ; p<0.01)$. It should also be noted that low-frequency bursts occasionally appeared among high-frequency bursts, but the opposite could not be observed, i.e., low-frequency bursting neurons were not seen to fire high-frequency bursts.

Burst length fell between 53 and $161 \mathrm{msec}$, with a peak in the $55-70 \mathrm{msec}$ range. The variability of burst length in a neuron was low, and it was not correlated with mean burst length. Thus, the occurrence of long bursts in the case of a short-burst neuron or vice versa was rare. The mean number of spikes in a burst was less variable in a cell but varied widely among neurons, falling in the range of 2.25-13, and it was not correlated with intraburst frequency or with burst length. However, the majority of neurons (14 of 18) fired two to six spikes in a burst. The two bursting PV-IR groups were not significantly different in any of the burst parameters.

Phase relationship of $P V-I R$ theta bursting cells to hippocampal theta activity. The relative phase (phase difference) of the septal unit and the hippocampal EEG signal was calculated by using the cross-wavelet phase values for 16 PV-IR neurons (Fig. 4). Based on Rao's spacing test, the phase distribution of PV-IR neurons was shown to be non-uniform. Individual PV-IR neurons showed unimodal phase preference, one population firing around the trough (180), with the other group preferring the 
peak (330) of the ongoing theta, resulting in a bimodal cumulative phase histogram of all PV-IR cells (Fig. 4). It was readily apparent that PV-IR neurons avoid the falling phase of the theta cycle. To separate the two peaks of the phase histogram, a mixture of two von Mises PDFs was fitted onto the data, and a goodness-of-fit measure was calculated (Bhattacharyya, 1943) that indicated a perfect fit (0.98). The mean angles of 178.7 and 328.4 of the resulting two groups with equal (eight) number of cells were statistically different (Watson's $F$ test at $p<0.001$ ). The tendency for clustering around the two preferred angles were further indicated by the concentration parameter $(\kappa)$, which was high in both groups (6.72 and 6.09).

Correlation between the phase preference and firing pattern of PV-IR neurons was also investigated. The proportion of long-burst neurons was somewhat higher in the positive peak-preferring group, whereas the opposite tendency (short bursts) was observed in the group concentrated around the trough, although this correlation was not significant (median and interquartile range, $94 \pm 45.2$ vs $65.8 \pm 55.7$ msec; $p=0.053 ; \chi^{2}$ test). No correlation was found between phase preference and firing pattern type (four constitutively bursting and four tail-pinchresponsive bursting in the troughpreferring group, and three and five in the peak-preferring group).

\section{Anatomy}

Identification of neurons by

double-fluorescent immunocytochemistry The majority of juxtacellularly labeled cells survived until perfusion (1-6 hr), and only a few of them were irreversibly damaged by the ionthophoresis or the electrode itself. Successfully labeled neurons had to fulfill several criteria for unequivocal identification. Their anatomical position in the medial septum had to match the expected position based on the stereotaxic coordinates registered during the electrophysiological session. If two or more cells became labeled, the recorded cell could be identified only if its labeling intensity and completeness of the arbors were far greater than that of the other cells. To avoid false-negative staining of filled cells attributable to weak penetration of the antibodies, their processes reaching the surface of the sections were checked for immunoreactivity (in the best cases, the cell bodies were cut in half) (Fig. 2, 250).

A total of 20 recorded neurons proved to be PV-IR neurons (Tables 1,2), of which eight belonged to the constitutively bursting (Fig. 2, 250), 10 to the tail-pinch-responsive bursting (Fig. 2, 258), and two to the tonic firing (Fig. 2, 359) groups. In relation to phase preference, eight preferred to fire at the trough and eight at the peak (Fig. 2, 250 and 258) of pyramidal layer theta.

B

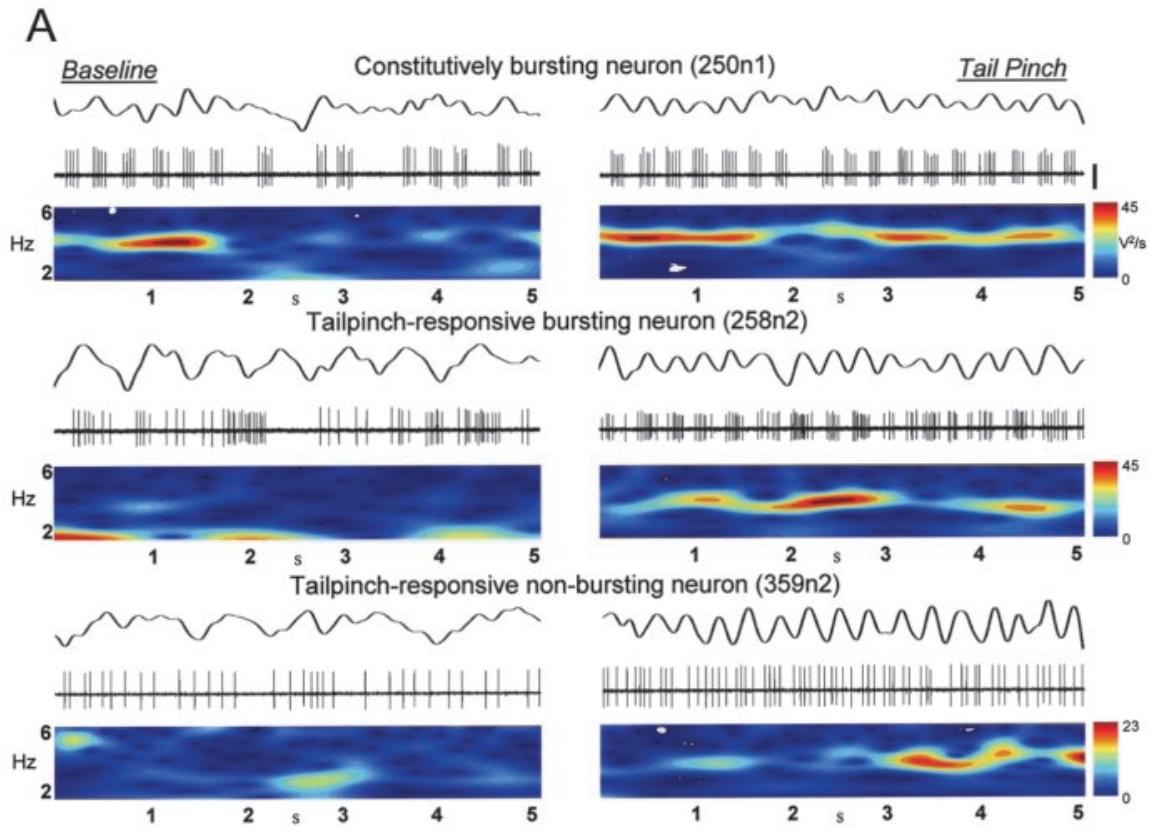

Inhibition (250n1)
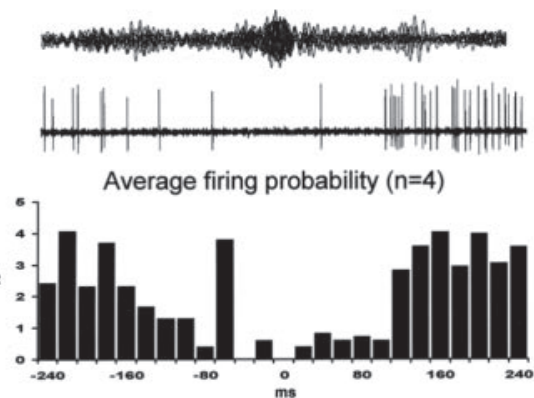

Activation (318n1)
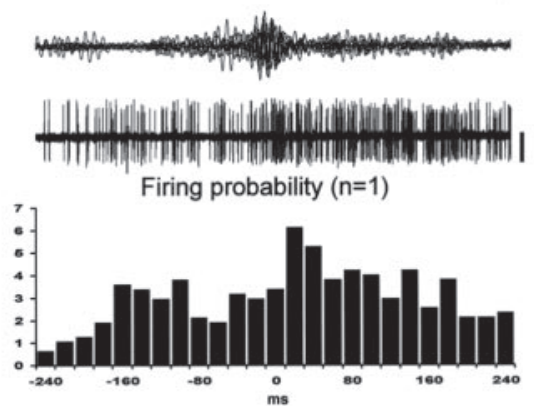

Figure 1. Firing pattern of PV-IR neurons during theta $(A)$ and high-frequency ripples $(B) . A$, Theta-related activity. Left column, baseline recordings, right column,tail-pinch recordings. Below the raw traces (filtered EEG and unit, respectively, in al (hit; see black vertical bar at the right of the topmost panel, right column, the unit recording of PV-IR neuron coded 250n1):

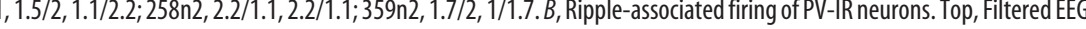
centered at the peak of the ripples. Bin size, $20 \mathrm{msec}$. Calibration in volts for the traces (shown as EEG/unit; see black vertical bar at right): $250 \mathrm{n} 1,0.5 / 1.75 ; 318 \mathrm{n} 1,0.65 / 2.2)$.

Reconstruction of parvalbumin-immunoreactive neurons

The post-labeling survival time of the animal had a major impact on the staining intensity and completeness of the dendritic and axonal arbors of the recorded and filled cells. If the perfusion was performed immediately after the labeling procedure, only the soma was recovered (Fig. 2, 359). Longer survival times allowed a detailed labeling of dendrites and axons but decreased the ratio of recovered neurons.

The constitutively bursting and tail-pinch-responsive bursting neurons expressed similar morphological characteristics. Two to four rarely branching dendrites emanated from a usually fusiform, sometimes round soma and arborized in a 600-800 $\mu \mathrm{m}$ diameter spherical volume. The axons of the reconstructed neurons emerged and ran in the rostroventral direction for sev- 

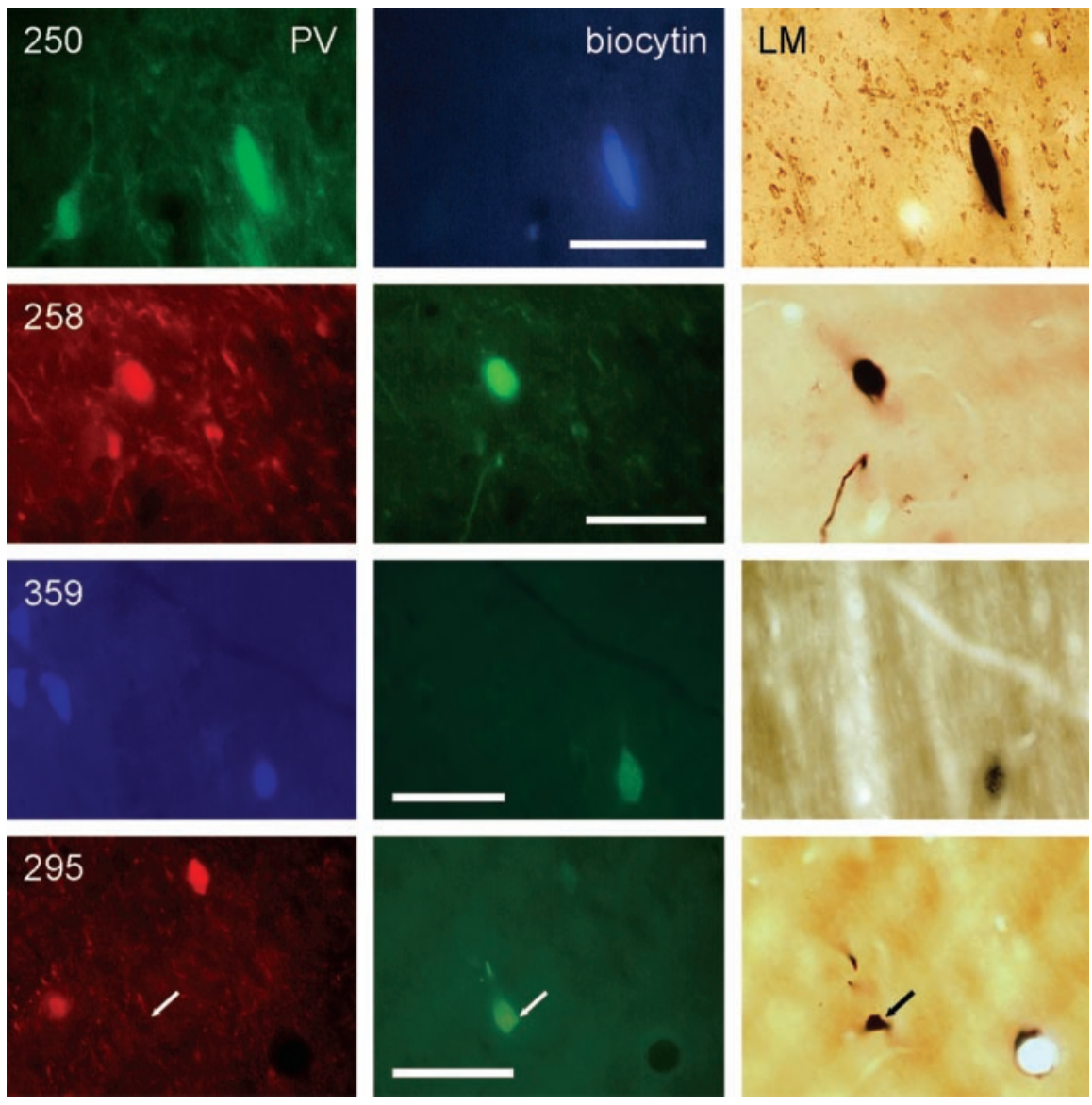

Figure 2. Identification of juxtacellularly labeled neurons. The cells from experiments (rows), numbered 250, 258, and 359 (constitutively bursting, tail-pinch-responsive bursting, and tonic firing neuron, respectively) are parvalbumin immunoreactive, whereas number 295 is PV negative (arrows), as shown in the fluorescent micrographs in the first column (PV) using different fluorochromes. The middle column indicates the biocytin (or Neurobiotin) labeling by fluorescent markers, and, in the right column, light micrographs of the same neurons are shown after nickel-intensified DAB reaction. Scale bars, $50 \mu \mathrm{m}$.

eral hundred micrometers and then turned dorsally (Fig. 5, wide arrows) to leave the medial septum in the dorsocaudal direction toward the fornix. The main axon emitted two different types of collaterals. The first type was thick myelinated secondary fibers that ran parallel with the main axon and left the medial septum dorsocaudally toward the hippocampus or rostroventrally, fading out in or after crossing the diagonal band of Broca, suggesting that these axons may target distant brain areas. The other type of local collaterals originated from the main axon or from the secondary myelinated axons. These unmyelinated fibers ran for several hundred micrometers in various directions, bearing irregularly spaced boutons. The density of these collaterals was the highest close to the soma, and, as the distance from the soma increased, these branches became fainter and impossible to follow. However, some short collateral segments were found in the horizontal limb of the diagonal band of Broca. A possible explanation is that neurobiotin diffuses or transports at a higher rate in thick axons, whereas the thin unmyelinated collaterals start to fill up later or more slowly. Labeling of the two tonic firing PV-IR neurons (Fig. 2, 359) was too weak for a detailed anatomical examination.

Morphological parameters of the recorded neurons were measured (minor and major axes and area of the soma and its distance from the midline), but no significant (Mann-Whitney $U$ test) correlation was found with physiological parameters such as firing pattern or phase.
Electron microscopic features of the labeled neurons

The completeness of labeling for a few neurons and the ultrastructure of the tissue allowed electron microscopic examination (Fig. 6). Continuously bursting PV-IR neurons (experiments 173 and 209) showed similar electron microscopic features in addition to their physiological behavior and light microscopic morphology. Symmetric synapses $(n=20$ from experiment 173; $n=20$ from experiment 209) were formed by either large-diameter axons filled with mitochondria $(n=13$ in both) or intervaricose segments (thin, without mithochondria; $n=6$ in both). The targets consisted of proximal dendrites $(n=11$ in both), middle-sized dendrites $(n=6,5)$, and distal dendrites $(n=$ $2,3)$. Symmetric synapses of the tailpinch-responsive neuron (experiment 258) were found on thick, proximal dendrites $(n=5)$, soma $(n=2)$ or distal dendrites $(n=2)$.

\section{Discussion}

The present study of PV-IR septal neurons revealed heterogeneity in firing properties in contrast to the remarkable consistency in phase relationship to hippocampal theta: they fired in one of two distinct phases, at the trough or the peak. The existence of different firing patterns and phase preferences (see below) of PV-IR neurons raises the question of whether the observed groups correspond to physiologically relevant categories or are simply generated by uncontrolled experimental conditions. The latter can be excluded because, in several experiments, neurons of different firing pattern and/or phase preference were recorded in the same animal.

The morphological variables that could be analyzed under the present experimental conditions revealed no correlation between morphological characteristics and firing pattern of neurons. In recent in vitro studies, remarkable physiological heterogeneity of PV-IR or GABAergic (GAD67 expressing) medial septal neurons was found: among GAD67 mRNA-containing GABAergic neurons, different firing modes could be observed (Sotty et al., 2003). In a recent work examining five electrophysiologically identified neuron types in the medial septum the putative PV-IR cells (fast firing and burst firing) could not be clearly distinguished morphologically (Morris et al., 1999). Furthermore, fast spiking, PV-IR neurons were morphologically homogenous, except for their axon terminal type [en passant or basket (Henderson et al., 2004)]. Based on the latter characteristics, two subgroups could be distinguished, but the physiological correlates of this morphological difference were not reported. The detailed morphological analysis of the axon arbor of different electrophysiological cell types of the MS revealed that classical, fast-spiking, PV-IR neurons show similar gross morphology as the neurons presented in this study: either constitutively bursting or tail-pinch-responsive bursting neurons (Henderson et al., 2001).

The firing pattern of a neuron is primarily determined by its 

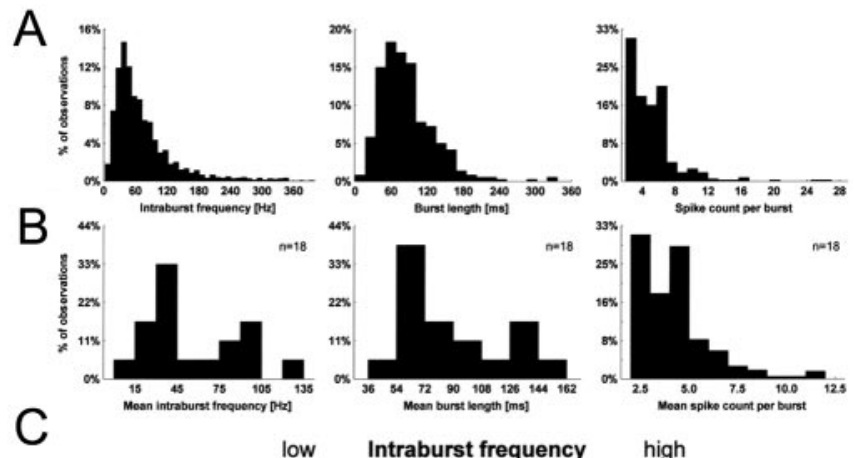

high

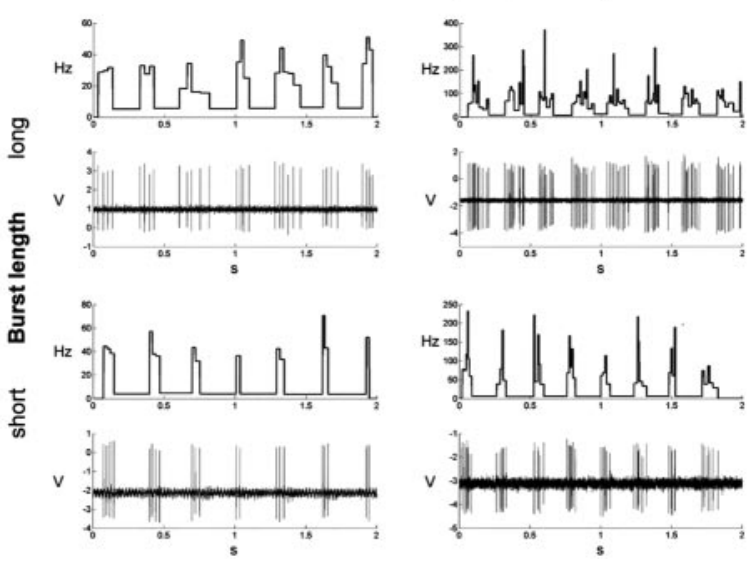

Figure 3. PV-IR neurons fired different types of bursts. $A$, Normalized (by total event count) distributions of the analyzed burst parameters are shown. From left to right, Intraburst frequency (bin, $10 \mathrm{~Hz}$ ), burst length (bin, $15 \mathrm{msec}$ ), and spike count per burst (bin, 1.5). $B$, The distribution of means of the above variables calculated for each cell is plotted. $C$, The different burst types can be seen. The top trace represents the instantaneous frequency function, and the bottom trace is the corresponding raw unit recording. Four burst types are demonstrated: (1) low-frequency, long burst; (2) low-frequency, short burst; (3) high-frequency, short burst; and (4) high-frequency, long burst. Transient increase in intraburst frequency can be observed within bursts, especially in case of high-frequency, long bursts. It accounts for the higher variability of intraburst frequency in the case of high-frequency bursts.

ion channel repertoire, whereas the timing of neuronal action potential generation to an ongoing field potential oscillation is primarily affected by the inputs of the given neuron. Thus, physiological deviations likely emerge from differences in ion channel expression patterns (Martina and Jonas, 1997; Martina et al., 1998) detected even in a morphologically-neurochemically homogenous cell population, as demonstrated in various brain regions, including the medial septum (Lenz et al., 1994; Chow et al., 1999; Tansey et al., 2002; Sotty et al., 2003). Both experimental and modeling studies have demonstrated that stable oscillatory activity can emerge in networks of heterogeneous inhibitory neurons. Furthermore, the stability of network oscillations increases with larger variation in physiological parameters (Aradi and Soltesz, 2002; Foldy et al., 2004). Our results, together with data showing remarkable heterogeneity in a given neurochemicalmorphological neuron type (Pawelzik et al., 2002; Monyer and Markram, 2004), raise the possibility that the variability in firing pattern can contribute to the stabilization of theta oscillation in the septal network, whereas phase difference may be determined from the interaction of mutually connected PV-IR neurons (see below).

The constitutively bursting PV-IR neurons exhibited hippocampus-independent theta bursting that became more regular during hippocampal theta. In previous studies, persistent
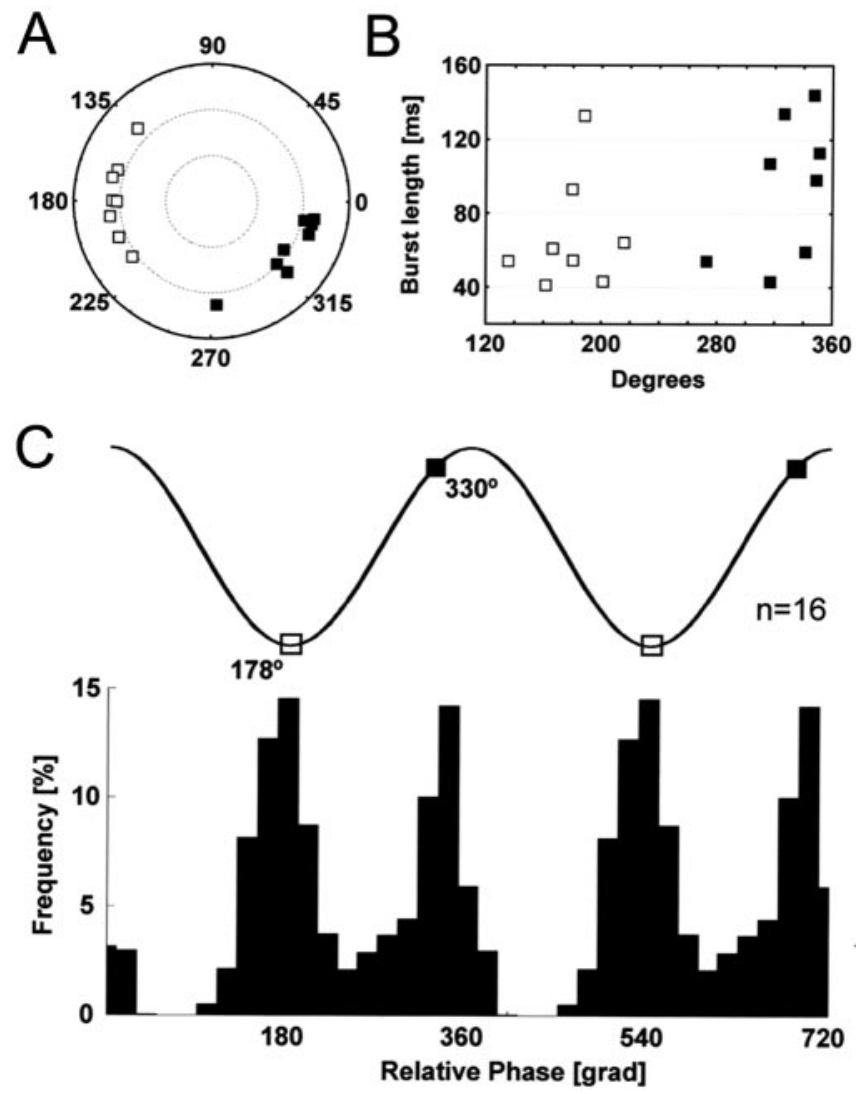

Figure 4. Phase preference and phase-firing pattern relationship of PV-IR neurons. $A$, The phase relationship of the two PV-IR groups ( $\square$ and $\square$ ) is shown on a polar plot. Strong phase preference is indicated by the distance from the center corresponding to the mean vector length (the plotted range: center, 0.3 ; periphery, 1.2). $B$, Weak correlation between burst length and phase preference of PV-IR neurons. Long-burst neurons tended to fire around the peak, whereas short-burst neurons fired around the trough of hippocampal theta. C, PV-IR neurons showed strong phase preference forming two well-separable groups. The two groups were significantly different from each other. In the top, two pyramidal layer theta cycles are shown. Below the theta cycles on the cumulative histograms, the phase distribution of PV-IR neurons (for details, see Materials and Methods) is demonstrated with mean phase values for all groups. PV-IR neurons have a bimodal phase distribution, exhibiting increased firing probability on the trough and slightly before the peak of pyramidal layer theta, and they avoid to fire on the falling edge of the cycle. For comparison, in previous papers (Klausberger et al., 2003), trough was $0^{\circ}$; in our calculation, trough was $180^{\circ}$.

bursting of unidentified septal neurons was observed in both anesthetized and freely moving animals (Ford et al., 1989; King et al., 1998). Furthermore, these neurons were characterized by subthreshold theta frequency membrane potential oscillations independent of hippocampal EEG activity (Barrenechea et al., 1995). In both these studies and our experiments, the appearance of hippocampal theta was accompanied by increased regularity of theta bursting, leading to tight coupling of theta bursting septal units and hippocampal EEG. The anatomical substrate of this synchronization of pacemaker units may also involve their local axon collateral networks revealed in the present study. Their axon arbor stretches to innervate the entire dorsoventral and rostrocaudal extent of the medial septum and the nucleus of the diagonal band of Broca complex (including the territory of PV-IR neurons), suggesting that they may play a role in synchronizing the activity of the entire septal network. A thick myelinated main axon that runs through the septum and emits short unmyelinated collaterals can transmit synchronous activity with minimal delay at long distance (Lang and Rosenbluth, 2003), even to other brain 


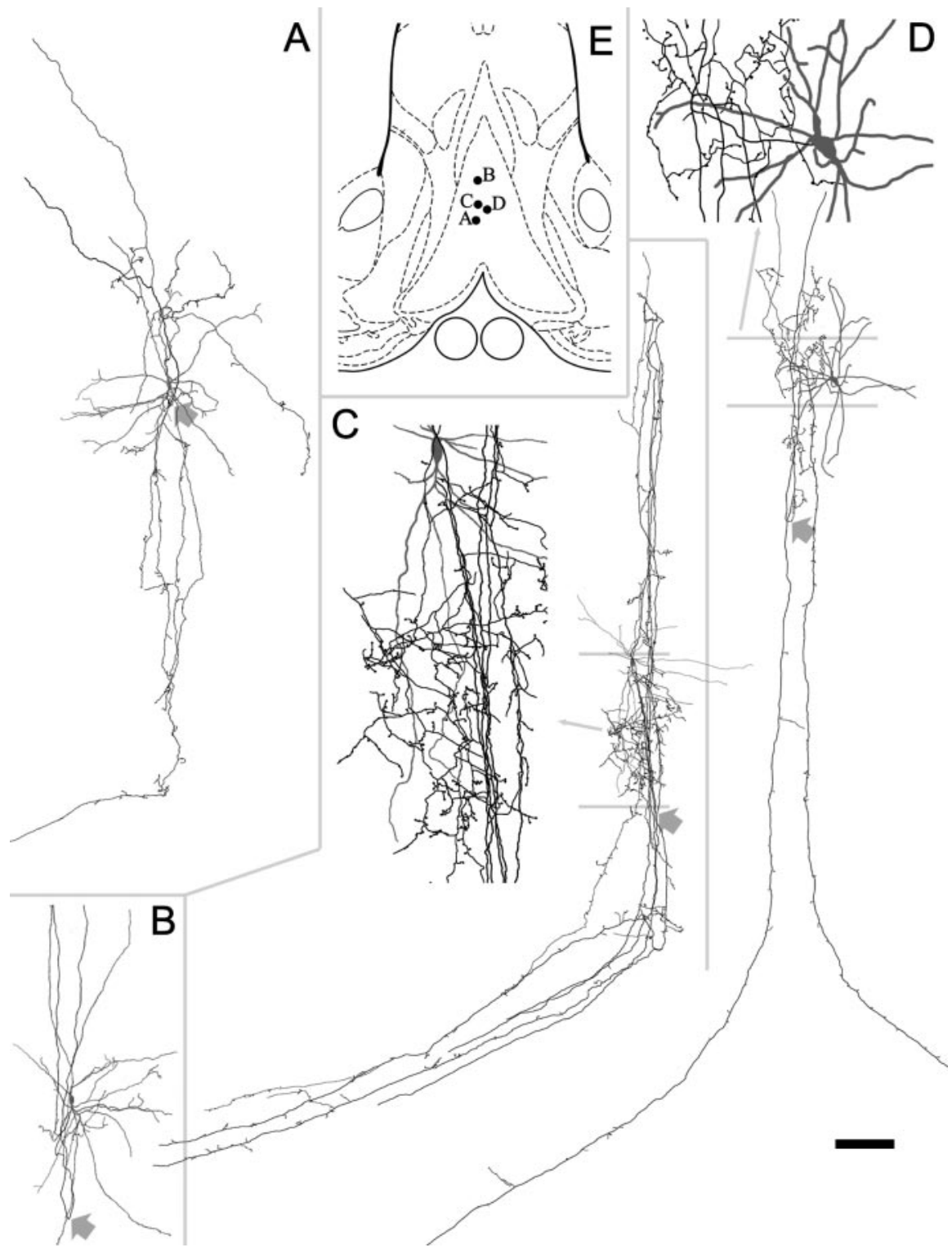

Figure 5. Reconstructions of four PV-IR, juxtacellularly filled neurons. The tail-pinch-responsive bursting $(A, 258)$ and the three constitutively bursting cells $(B, 250 ; C, 209 ; D, 173)$ show common morphological features, despite the differences in the extent of their labeling: dendritic arbor in an oval-shaped area (gray), a thick main axon emerging ventrally and taking a U-turn (wide gray arrows), leaving the septum dorsally. This main axon emits parallel-running, thick myelinated secondary fibers that leave the medial septum dorsally with the main axon or ventrally by entering or crossing the diagonal band of Broca. The thick myelinated axons emit thin unmyelinated collaterals bearing the terminals ( $3 \times$ magnified area in $C, D$ ). Scale bar: $200 \mu \mathrm{m}$; for the magnified insets, $66 \mu \mathrm{m}$.

regions. This is supported by our finding that projecting axons of PV-IR neurons leave the septal complex in two main directions, possibly forming a dorsal and a ventral route toward limbic cortical (hippocampus, entorhinal, perirhinal, retrosplenial, and anterior cingulated cortices) and subcortical regions (Meibach and Siegel, 1977; Gaykema et al., 1990).

The tail-pinch-responsive bursting neurons may be switched to bursting mode by the synchronized rhythmic input of constitutively bursting neurons in conjunction with the ascending activation and hippocampal feedback, which synchronizes both these and constitutively bursting cells. Activation can be conveyed by excitatory input from the hypothalamus and brainstem (Vertes, 1988; Borhegyi et al., 1998; Kiss et al., 2000), which may result in the elevation of local GABAergic tone. The latter may be supported by our finding that a small population of PV-IR GABAergic neurons termed here as tonic theta $\mathrm{ON}$ cells, robustly increases its firing rate at the onset of hippocampal theta.

The hippocampo-septal GABAergic projection targeting mainly PV-IR neurons may also contribute to the synchronization of PV-IR pacemaker units (Toth et al., 1993; Wang, 2002). Stratum oriens neurons with long horizontal dendrites (likely including the hippocampo-septal GABAergic neurons) collect inputs from a large number of pyramidal cells (Blasco-Ibanez and Freund, 1995). Synchronized activity of some of these converging pyramidal cells may activate hippocampo-septal neurons, which, in turn, can align and phase lock the pacemaker units in the MS.

The intraburst frequency of most PV-IR neurons fell in the gamma range (Serafin et al., 1996). Consequently, PV-IR neurons can induce gamma bursts in postsynaptic cells. Likewise, several hippocampal interneuron classes fire gamma frequency bursts or can resonate to gamma frequency inputs (Pike et al., 2000; Klausberger et al., 2003). Furthermore, gamma frequency oscillations in the hippocampus appear embedded in theta activity (Bragin et al., 1995).

Sharp wave-associated inhibition was detected in the majority of bursting PV-IR neurons (that could be tested), and this inhibition out-spanned the duration of ripples, as determined by previously established criteria (for details, see Materials and Methods). Inhibition can be caused by the hippocampo-septal GABAergic projection innervating PV-IR cells in the medial septum (Toth et al., 1993). During the synchronization process, the hippocampo-septal neurons might be activated before pyramidal cell synchronous firing reaches the level of a detectable ripple. Thus, the beginning of the inhibitory response precedes the onset of ripples, unless the onset of ripples is redefined (e.g., to commence with the activation of hippocampo-septal inhibition).

PV-IR neurons formed two groups based on phase relationship to the ongoing hippocampal theta: a trough-preferring group and a peak-preferring group $\left(178^{\circ}\right.$ and $330^{\circ}$, respectively). These two phases match data from unidentified rhythmic medial septal neurons obtained in freely moving animals (Dragoi et al., 1999). The local axon collateral network of PV-IR neurons, which overlaps with the territory defined by the distribution of PV-IR neuronal elements, suggests that these neurons are likely to be interconnected. The detected asymmetry in the division of the theta cycle by the two PV-IR phase groups correlates (almost significantly; see Results) with the difference in their burst length. This observation, together with the anatomical data, raises the 

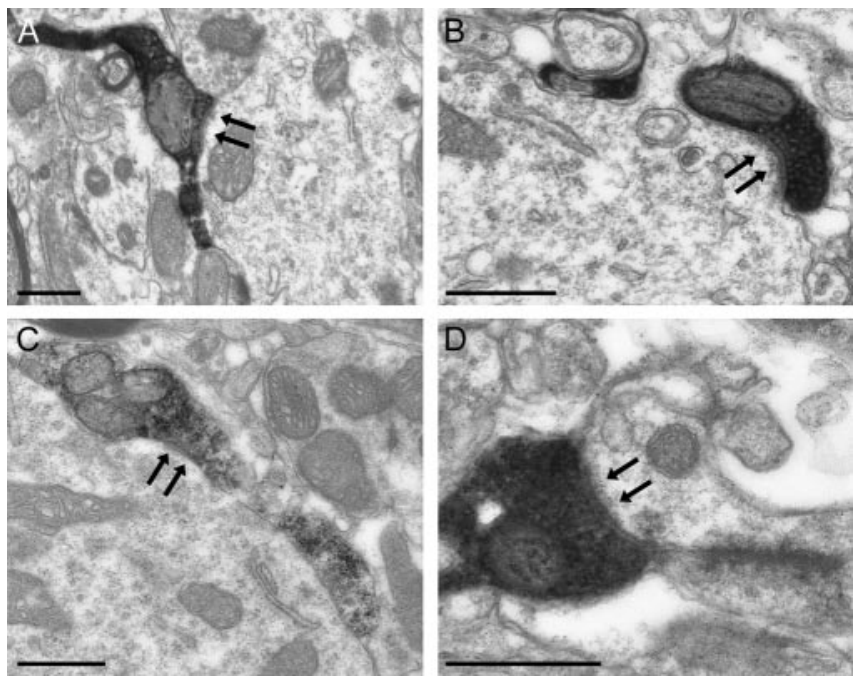

Figure 6. Electron microscopic features of the biocytin- or Neurobiotin-filled axons. The terminals of constitutively or theta-responsive bursting, PV-IR neurons formed symmetrical synapses (double arrows) frequently on proximal (thick) or middle-sized dendrites $(A, B) .0 C$ casionally, somata ( $C$ or thin, distal dendrites $(D)$ were also targeted. Scale bars, $500 \mathrm{~nm}$.
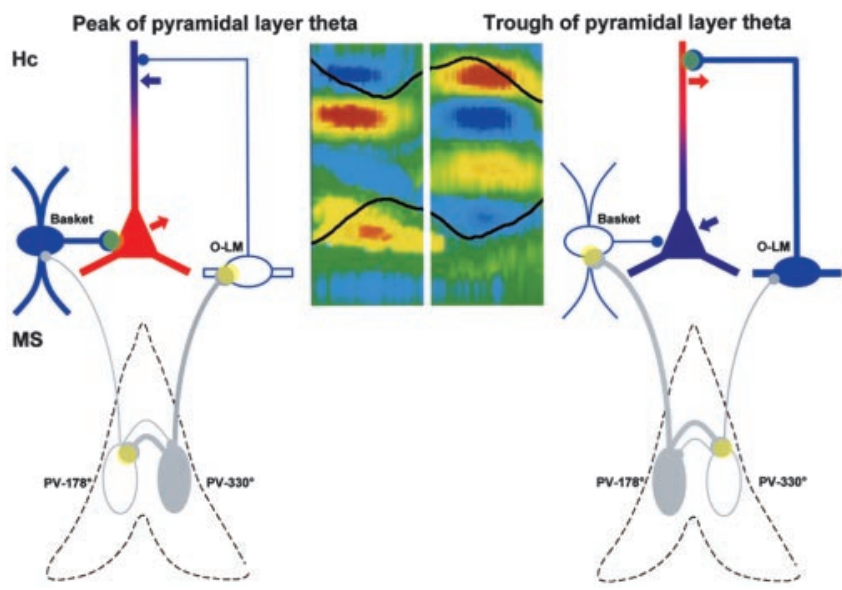

Figure 7. A hypothetical scheme showing the interaction between medial septal PV-IR neurons and hippocampal perisomatic and dendritic inhibitory interneurons during the peak (left) and the trough (right) of pyramidal layer theta. The corresponding current source density maps (with permission from G. Buzsaki, 2002) and EEG recordings are also shown. The bottom EEG trace derives from the pyramidal layer, and the top trace is from the stratum lacunosummoleculare. Left, The peak-preferring PV-IR cells of the medial septum are firing and inhibiting both the trough-preferring PV-IR neurons of the MS and dendritic inhibitory interneurons in the hippocampus, resulting in disinhibition of the distal dendritic region and the appearance of a marked current sink in the lacunosum-moleculare on the current source density map. Basket cells are active in this phase and induce a source in stratum pyramidale. Right, The troughpreferring neurons are active and suppress both the peak-preferring MS neurons and hippocampal perisomatic inhibitory cells. As a result, the basket cell-mediated active source disappears in the stratum pyramidale, and a new source (likely active) appears in stratum lacunosum-moleculare because of the activity of 0 -LM cells.

possibility that the local connections of PV-IR neurons may be segregated, i.e., the two phase groups may preferentially innervate each other, thereby influencing their phase relationship to hippocampal theta. In addition, the phase preference of PV-IR neurons is also influenced by supramammillary (Kocsis and Vertes, 1994) and hippocampal (Wang, 2002) inputs.

The bimodal phase preference of PV-IR cells is in good agreement with the well known phase shift through the layers of the hippocampus (Buzsaki et al., 1986) and the recently described phase preference of perisomatic and dendritic inhibitory neurons (Klausberger et al., 2003). The phase difference between the pyramidal layer and hippocampal fissure is $\sim 180^{\circ}$, close to the $150^{\circ}$ observed between the two PV-IR cell groups in our experiments. Around the trough of pyramidal layer theta, the firing probability of pyramidal neurons is the highest (Klausberger et al., 2003). It is supposed to be the result of disinhibition brought about by the septo-hippocampal GABAergic connection (Toth et al., 1997). With a half-cycle later, the maximal level of depolarization can be detected in the hippocampal fissure coincident with perisomatic inhibition (Kamondi et al., 1998). Dendritic interneurons, similar to perisomatic inhibitory cells, are heavily innervated by septal afferents (Freund and Antal, 1988; Gulyas et al., 1990, 1991), which, together with our results, may imply that the source of enhanced dendritic depolarization can partly be attributable to disinhibition by septo-hippocampal inputs. Indeed, recent experiments (Klausberger et al., 2003) demonstrated that perisomatic (PV-positive basket cells) and dendritic (O-LM cells containing somatostatin) inhibitory neurons fire at different phases of theta, the latter together with pyramidal cells at the trough and the former around the peak. Accordingly, we hypothesize (Fig. 7) that the two PV-IR phase groups have distinct hippocampal targets: the trough-preferring cells innervate perisomatic inhibitory neurons, whereas the positive peak-preferring neurons target dendritic inhibition.

\section{References}

Aradi I, Soltesz I (2002) Modulation of network behaviour by changes in variance in interneuronal properties. J Physiol (Lond) 538:227-251.

Barrenechea C, Pedemonte M, Nunez A, Garcia-Austt E (1995) In vivo intracellular recordings of medial septal and diagonal band of Broca neurons: relationships with theta rhythm. Exp Brain Res 103:31-40.

Batschelet E (1981) Circular statistics in biology. London: Academic.

Bhattacharyya A (1943) On a measure of divergence between two statistical populations defined by their probability distributions. Bull Calcutta Math Soc 35:99-110.

Blasco-Ibanez JM, Freund TF (1995) Synaptic input of horizontal interneurons in stratum oriens of the hippocampal CA1 subfield: structural basis of feed-back activation. Eur J Neurosci 7:2170-2180.

Borhegyi Z, Magloczky Z, Acsady L, Freund TF (1998) The supramammillary nucleus innervates cholinergic and GABAergic neurons in the medial septum-diagonal band of Broca complex. Neuroscience 82:1053-1065.

Bragin A, Jando G, Nadasdy Z, Hetke J, Wise K, Buzsaki G (1995) Gamma $(40-100 \mathrm{~Hz})$ oscillation in the hippocampus of the behaving rat. J Neurosci 15:47-60.

Brazhnik ES, Fox SE (1999) Action potentials and relations to the theta rhythm of medial septal neurons in vivo. Exp Brain Res 127:244-258.

Buzsaki G (2002) Theta oscillations in the hippocampus. Neuron 33:325-340.

Buzsaki G, Leung LW, Vanderwolf CH (1983) Cellular bases of hippocampal EEG in the behaving rat. Brain Res 287:139-171.

Buzsaki G, Czopf J, Kondakor I, Kellenyi L (1986) Laminar distribution of hippocampal rhythmic slow activity (RSA) in the behaving rat: currentsource density analysis, effects of urethane and atropine. Brain Res 365:125-137.

Chow A, Erisir A, Farb C, Nadal MS, Ozaita A, Lau D, Welker E, Rudy B (1999) $\mathrm{K}^{+}$channel expression distinguishes subpopulations of parvalbumin- and somatostatin-containing neocortical interneurons. J Neurosci 19:9332-9345.

Cocatre-Zilgien JH, Delcomyn F (1992) Identification of bursts in spike trains. J Neurosci Methods 41:19-30.

Dekhuijzen AJ, Bagust J (1996) Analysis of neural bursting: nonrhythmic and rhythmic activity in isolated spinal cord. J Neurosci Methods 67:141-147.

Dragoi G, Carpi D, Recce M, Csicsvari J, Buzsaki G (1999) Interactions between hippocampus and medial septum during sharp waves and theta oscillation in the behaving rat. J Neurosci 19:6191-6199.

Foldy C, Aradi I, Howard A, Soltesz I (2004) Diversity beyond variance: 
modulation of firing rates and network coherence by GABAergic subpopulations. Eur J Neurosci 19:119-130.

Ford RD, Colom LV, Bland BH (1989) The classification of medial septumdiagonal band cells as theta-on or theta-off in relation to hippocampal EEG states. Brain Res 493:269-282.

Freund TF (1989) GABAergic septohippocampal neurons contain parvalbumin. Brain Res 478:375-381.

Freund TF, Antal M (1988) GABA-containing neurons in the septum control inhibitory interneurons in the hippocampus. Nature 336:170-173.

Frotscher M, Leranth C (1985) Cholinergic innervation of the rat hippocampus as revealed by choline acetyltransferase immunocytochemistry: a combined light and electron microscopic study. J Comp Neurol 239:237-246.

Gaykema RP, Luiten PG, Nyakas C, Traber J (1990) Cortical projection patterns of the medial septum-diagonal band complex. J Comp Neurol 293:103-124.

Gaztelu JM, Buno Jr W (1982) Septo-hippocampal relationships during EEG theta rhythm. Electroencephalogr Clin Neurophysiol 54:375-387.

Gogolak G, Stumpf C, Petsche H, Sterc J (1968) The firing pattern of septal neurons and the form of the hippocampal theta wave. Brain Res 7:201-207.

Gulyas AI, Gorcs TJ, Freund TF (1990) Innervation of different peptidecontaining neurons in the hippocampus by GABAergic septal afferents. Neuroscience 37:31-44.

Gulyas AI, Seress L, Toth K, Acsady L, Antal M, Freund TF (1991) Septal GABAergic neurons innervate inhibitory interneurons in the hippocampus of the macaque monkey. Neuroscience 41:381-390.

Henderson Z, Morris NP, Grimwood P, Fiddler G, Yang HW, Appenteng K (2001) Morphology of local axon collaterals of electrophysiologically characterised neurons in the rat medial septal/ diagonal band complex. J Comp Neurol 430:410-432.

Henderson Z, Fiddler G, Saha S, Boros A, Halasy K (2004) A parvalbumincontaining, axosomatic synaptic network in the rat medial septum: relevance to rhythmogenesis. Eur J Neurosci 19:2753-2768.

Kamondi A, Acsady L, Wang XJ, Buzsaki G (1998) Theta oscillations in somata and dendrites of hippocampal pyramidal cells in vivo: activitydependent phase-precession of action potentials. Hippocampus 8:244-261.

King C, Recce M, O'Keefe J (1998) The rhythmicity of cells of the medial septum/diagonal band of Broca in the awake freely moving rat: relationships with behaviour and hippocampal theta. Eur J Neurosci 10:464-477.

Kiss J, Csaki A, Bokor H, Shanabrough M, Leranth C (2000) The supramammillo-hippocampal and supramammillo-septal glutamatergic/aspartatergic projections in the rat: a combined $\left[{ }^{3} \mathrm{H}\right] \mathrm{D}$-aspartate autoradiographic and immunohistochemical study. Neuroscience 97:657-669.

Klausberger T, Magill PJ, Marton LF, Roberts JD, Cobden PM, Buzsaki G, Somogyi P (2003) Brain-state- and cell-type-specific firing of hippocampal interneurons in vivo. Nature 421:844-848.

Kocsis B, Vertes RP (1994) Characterization of neurons of the supramammillary nucleus and mammillary body that discharge rhythmically with the hippocampal theta rhythm in the rat. J Neurosci 14:7040-7052.

Lang EJ, Rosenbluth J (2003) Role of myelination in the development of a uniform olivocerebellar conduction time. J Neurophysiol 89:2259-2270.

Lenz S, Perney TM, Qin Y, Robbins E, Chesselet MF (1994) GABA-ergic interneurons of the striatum express the Shaw-like potassium channel Kv3.1. Synapse 18:55-66.

Leung LW (1985) Spectral analysis of hippocampal EEG in the freely moving rat: effects of centrally active drugs and relations to evoked potentials. Electroencephalogr Clin Neurophysiol 60:65-77.

Mardia KV, Jupp PE (2000) Directional statistics. Chichester, UK: Wiley.
Martina M, Jonas P (1997) Functional differences in $\mathrm{Na}^{+}$channel gating between fast-spiking interneurones and principal neurones of rat hippocampus. J Physiol (Lond) 505:593-603.

Martina M, Schultz JH, Ehmke H, Monyer H, Jonas P (1998) Functional and molecular differences between voltage-gated $\mathrm{K}^{+}$channels of fastspiking interneurons and pyramidal neurons of rat hippocampus. J Neurosci 18:8111-8125.

Meibach RC, Siegel A (1977) Efferent connections of the septal area in the rat: an analysis utilizing retrograde and anterograde transport methods. Brain Res 119:1-20.

Monyer H, Markram H (2004) Interneuron diversity series: molecular and genetic tools to study GABAergic interneuron diversity and function. Trends Neurosci 27:90-97.

Morris NP, Harris SJ, Henderson Z (1999) Parvalbumin-immunoreactive, fast-spiking neurons in the medial septum/diagonal band complex of the rat: intracellular recordings in vitro. Neuroscience 92:589-600.

Muthuswamy J, Thakor NV (1998) Spectral analysis methods for neurological signals. J Neurosci Methods 83:1-14.

Pawelzik H, Hughes DI, Thomson AM (2002) Physiological and morphological diversity of immunocytochemically defined parvalbumin- and cholecystokinin-positive interneurones in CA1 of the adult rat hippocampus. J Comp Neurol 443:346-367.

Pike FG, Goddard RS, Suckling JM, Ganter P, Kasthuri N, Paulsen O (2000) Distinct frequency preferences of different types of rat hippocampal neurones in response to oscillatory input currents. J Physiol (Lond) 529:205-213.

Pinault D (1996) A novel single-cell staining procedure performed in vivo under electrophysiological control: morpho-functional features of juxtacellularly labeled thalamic cells and other central neurons with biocytin or Neurobiotin. J Neurosci Methods 65:113-136.

Rao JS (1976) Some tests based on arc-lengths for a circle. Sankhya: The Indian J Stat B 4:329-338.

Serafin M, Williams S, Khateb A, Fort P, Muhlethaler M (1996) Rhythmic firing of medial septum non-cholinergic neurons. Neuroscience 75:671-675.

Sotty F, Danik M, Manseau F, Laplante F, Quirion R, Williams S (2003) Distinct electrophysiological properties of glutamatergic, cholinergic and GABAergic rat septohippocampal neurons: novel implications for hippocampal rhythmicity. J Physiol (Lond) 551:927-943.

Squire LR (1992) Memory and the hippocampus: a synthesis from findings with rats, monkeys, and humans. Psychol Rev 99:195-231.

Szucs A (1998) Applications of the spike density function in analysis of neuronal firing patterns. J Neurosci Methods 81:159-167.

Tansey EP, Chow A, Rudy B, McBain CJ (2002) Developmental expression of potassium-channel subunit Kv3.2 within subpopulations of mouse hippocampal inhibitory interneurons. Hippocampus 12:137-148.

Toth K, Borhegyi Z, Freund TF (1993) Postsynaptic targets of GABAergic hippocampal neurons in the medial septum-diagonal band of broca complex. J Neurosci 13:3712-3724.

Toth K, Freund TF, Miles R (1997) Disinhibition of rat hippocampal pyramidal cells by GABAergic afferents from the septum. J Physiol (Lond) 500:463-474.

Vertes RP (1988) Brainstem afferents to the basal forebrain in the rat. Neuroscience 24:907-935.

Vertes RP, Kocsis B (1997) Brainstem-diencephalo-septohippocampal systems controlling the theta rhythm of the hippocampus. Neuroscience 81:893-926.

Wang XJ (2002) Pacemaker neurons for the theta rhythm and their synchronization in the septohippocampal reciprocal loop. J Neurophysiol 87:889-900.

Zar JH (1996) Biostatistical analysis. Upper Saddle River, NJ: Prentice Hall. 